\title{
Antibody-drug conjugates-an emerging class of cancer treatment
}

\author{
Nikolaos Diamantis ${ }^{1}$ and Udai Banerji ${ }^{*}, 1$ \\ ${ }^{1}$ Drug Development Unit, The Institute of Cancer Research and The Royal Marsden, Downs Road, Sutton, London SM2 5PT, UK
}

Antibody-drug conjugates (ADCs) are an emerging novel class of anticancer treatment agents that combines the selectivity of targeted treatment with the cytotoxic potency of chemotherapy drugs. New linker technology associated with novel highly potent cytotoxic payloads has permitted the development of more effective and safe ADCs. In recent years, two ADCs have been licensed, T-DM1 and brentuximab vedotin, and are already establishing their place in cancer treatment. A plethora of ADCs are being investigated in phases I and II trials, emerging data of which appears promising. As we deepen our understanding of what makes a successful ADC, an increasing number of ADCs will likely become viable treatment options as single agents or in combination with chemotherapy. This review will present the philosophy underlying ADCs, their main characteristics and current research developments with a focus on ADCs in solid tumours.

Paul Ehrlich's vision of a rational targeted strategy against invading microbes or malignant cells has been a driving research throughout the past century, bringing a scientific and therapeutic revolution in cancer treatment. Although antibody-drug conjugates (ADCs) have been under investigation for decades, it is only recently with the advent of significant advances in engineering new linker and conjugation technologies together with highly potent cytotoxic drugs that we have realised the ADC's true potential (Panowksi et al, 2014). Monoclonal antibodies have proved to have an important role in cancer treatment with drugs such as trastuzumab, pertuzumab, cetuximab and rituximab becoming the standard of care in selected solid tumours and lymphomas. Classic chemotherapy, the mainstay of anticancer treatment, demonstrates limited selectivity against cancer cells leading to a small therapeutic window, thus limiting its efficacy.

Antibody-drug conjugates could bring these two classes of drugs with their complementing properties together, in creating a highly selective and highly cytotoxic cancer treatment with an increased therapeutic window, as envisaged by Paul Ehrlich (Hughes, 2010). Looking back into the reasons for previous failures gives us an opportunity to identify the crucial characteristics that will make ADC an effective anticancer treatment. The low chemotherapy drug potency, unstable linkers and low antigen selectivity are the most commonly identified weaknesses limiting the efficacy of an ADC (Perez et al, 2014). In addition, in some of the first clinical trials murine antibodies were used leading to high rates of immunogenicity and, therefore, to low efficacy (Hughes, 2010).
Antibody-drug conjugates have a complex structure with many moving parts, each of which has different properties and desirable characteristics. An ADC can be divided into three main structural units: the antibody, the cytotoxic agent and the linker, which will be discussed below.

\section{ANTIBODY-DRUG CONJUGATES}

Target antigen. The ideal target antigen should be: (a) highly expressed with limited heterogeneity across the tumour and with low normal tissue expression, for example, T-DM1 targets HER-2, a receptor that reaches levels of expression of $2 \times 10^{6}$ on HER-2positive cancer cells compared with $2 \times 10^{4}$ on other cells (ShefetCarasso and Benhar, 2015); (b) there should be minimal antigen shedding to prevent the antibody binding to its target within the circulation; and (c) the antibody should be well internalised by receptor-mediated endocytosis and should not be modulated during endocytosis. The target antigen should not be downregulated after treatment with the ADC (Mack et al, 2014). The minimum threshold of the different variables that are required to a make a tumour antigen an effective target is still undetermined and interdependent. Studies in lymphoma and prostate cancer have shown that a minimum value of tumour-antigen density is a prerequisite for ADC efficacy. The biomarker analysis study for TDM1 showed that although it was active across different HER-2 expression subgroups, patients with tumours who

*Correspondence: Dr U Banerji; E-mail: udai.banerji@icr.ac.uk

Received 14 July 2015; revised 16 October 2015; accepted 26 October 2015; published online 7 January 2016

(c) 2016 Cancer Research UK. All rights reserved 0007-0920/16

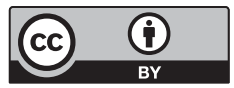


expressed HER-2 more highly derived the greatest benefit (Baselga et al, 2013). The desirable cutoff value of antigen expression varies greatly and depends on other target antigen properties such as the internalisation rate and binding affinity, as well as other ADC characteristics, such as cytotoxic payload and linker stability. There is evidence that ADCs can be effective even when they target antigens with low expression given minimal normal tissue expression (Perez et al, 2014). The problem of non-homogeneous expression of the target antigen in solid tumours could potentially be addressed by the bystander effect, that is, the process by which membrane-permeable free cytotoxic payload is able to induce cell death to the neighbouring cells after being internalised and cleaved from the linker. Conversely, the bystander effect can increase the ADCs off-target systemic toxicity. Various factors can affect the rate of internalisation of the ADC in the cancer cell, which is a poorly understood process. One such factor is the epitope on the target antigen. For example, different epitopes of the HER-2 receptor have resulted in significantly different rates of internalisation and degradation of the $\mathrm{mAb}-\mathrm{Ag}$ molecule. In addition, there are other difficulties while targeting cancer cell surface antigens, such as the high interstitial tumour pressure, downregulation of the antigen and the presence of other physical and kinetic barriers that diminish the cytotoxic payload uptake (Mack et al, 2014; Perez et al, 2014). Although most licensed or at an advanced research stage ADCs target tumour antigens, alternative approaches are actively being investigated to overcome some of the above mentioned limitations (Casi and Neri, 2012).

Another approach is to target antigens in stroma and vasculature. There is evidence in the preclinical and clinical setting that components of the neovasculature subendothelial extracellular matrix and of the tumour stroma could be valuable target antigens. A characteristic example is the extra-domain B (ED-B) of fibronectin which is a marker of angiogenesis, specifically highly expressed in vasculature of aggressive solid tumours (Palumbo et al, 2011) (Figure 1). The human mAb L19 which targets the ED$\mathrm{B}$ has been combined with different effector molecules and has been studied extensively as a potential ADC. This target was successfully exploited by conjugating it with tubulin inhibitors showing that non-internalising vascular targeting ADCs could offer other treatment approaches (Perrino et al, 2014), (Table 1).

A further interesting approach to targeting antigens is to target the tumour-initiating cells or cancer stem cells (TICs). The hypothesis is that since TICs comprise an aggressive sub-population of tumour cells that are responsible for tumour growth, metastasis and recurrence, targeting these cells will have a big impact on the disease control (Visvader and Lindeman, 2008). Sapra et al (2013) have developed an ADC combined with a tubulin inhibitor that targets 5T4 oncofetal antigen, which is an antigen expressed in TICs in NSCLC and has been associated with an invasive phenotype. A phase I trial investigating this ADC is currently recruiting patients with NSCLC and other solid tumours (Figure 1).

Antibody selection. Antibody engineering has made breakthroughs over recent years making it feasible to produce humanised and fully human antibodies as the basic components of ADCs. The early generation ADCs used murine antibodies causing significant immunogenicity, with many patients producing human anti-mouse antibodies thus reducing the efficacy of treatment.

The most commonly used antibody format currently is human IgG isotypes and in particular IgG1 (Hughes, 2010; Perez et al, 2014). The antibodies once part of the ADC can retain their original properties and activate immune functions such as antibodydependent cellular cytotoxicity (ADCC) or complement-dependent cytotoxicity (Perez et al, 2014). They could still act as receptor inhibitors or signal modulators. One example of complementary action is T-DM1 whose Ab's retain effector functions have been shown to activate ADCC (Junttila et al, 2011). The independent function of the antibody is not always beneficial and complementary to $\mathrm{ADC}$ efficacy, especially when $\mathrm{Ab}$ binding is sufficient to produce a cytotoxic effect. The Ab's independent effector functions could lead to increased toxicity, reduced tumour localisation and internalisation of the ADC (Sapra and Shor, 2013).

Linkers and conjugation. The linker plays a crucial role since its properties greatly influence the ADC's pharmacokinetics, therapeutic index and efficacy (Hughes, 2010; Flygare et al, 2013; Shefet-Carasso and Benhar, 2015). The ideal linker should be stable so that the ADC does not release the cytotoxic drug before reaching its target and causing off-target toxicity. At the same time it should be able to release the drug efficiently once internalised (Teicher and Chari, 2011).

Another important factor is how many of the drug molecules will be loaded onto the antibody: the drug-antibody ratio (DAR). Attaching too few of the drug molecules will lead to decreased efficacy. Attach too many and the ADC will become unstable with altered pharmacokinetic properties, increased plasma clearance, reduced half-life and increased systemic toxicity (Perez et al, 2014). The currently licensed ADCs with proven activity are produced by nonspecific conjugation to lysine residues and to some degree

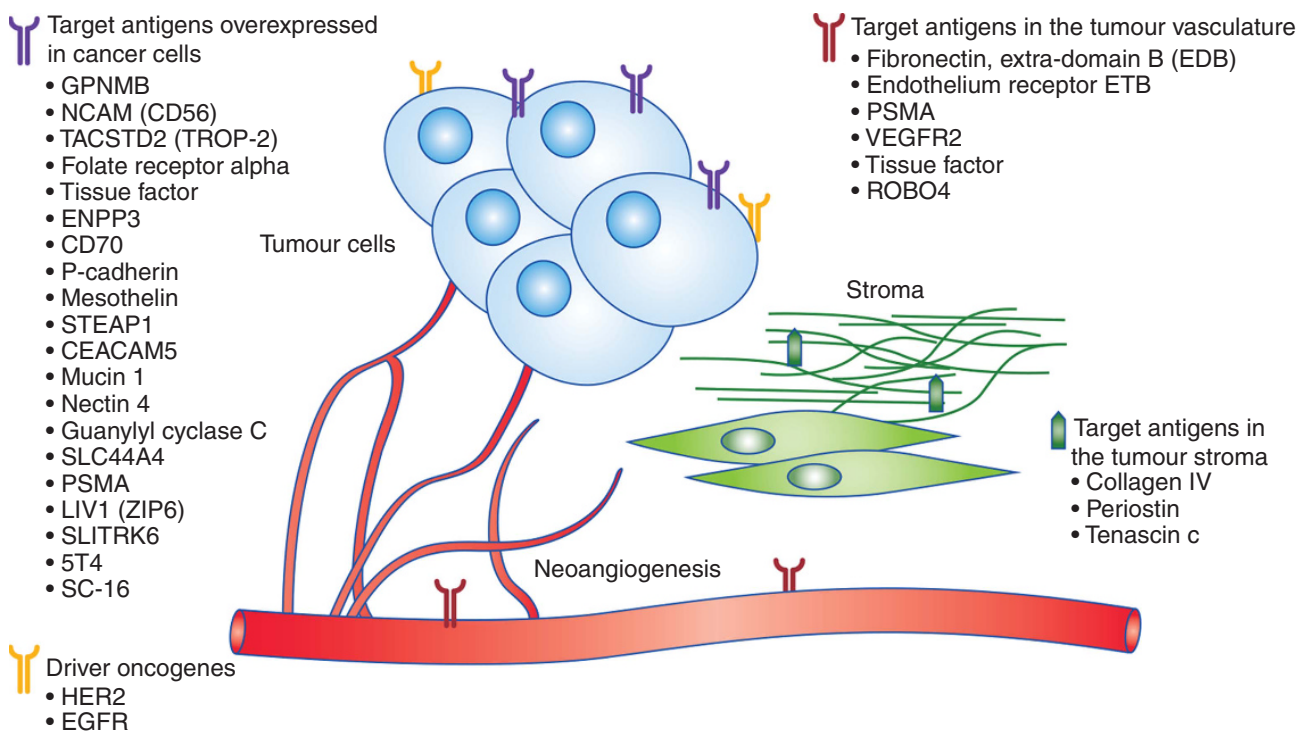

Figure 1. Target antigens for ADCs in solid tumours. 
Table 1. Target antigens in solid tumours under research

\begin{tabular}{|c|c|c|}
\hline Name & ADC & Lead \\
\hline \multicolumn{3}{|c|}{ Target antigens overexpressed in cancer cells } \\
\hline GPNMB & Glembatumumab vedotin [1] & Breast cancer and melanoma \\
\hline CD56 & Lorvotuzumab mertansine (IMGN-901) [2] & SCLC \\
\hline TACSTD2 (TROP2) & sacituzumab govitecan (IMMU-132) [3] & TNBC and pancreatic cancer \\
\hline CEACAM5 & Labetuzumab SN-38 [4] & Colorectal cancer \\
\hline Folate receptor- $\alpha$ & $\begin{array}{l}\text { - Mirvetuximab soravtansine (IMGN-853) [5] } \\
\text { - Vintafolide [6] }\end{array}$ & Ovarian and endometrial can \\
\hline Mucin 1 (Sialoglycotope CA6) & SAR-566658 [7] & Breast, ovarian, cervical, lung \\
\hline STEAP1 & Vandortuzumab vedotin RG-7450 [8] & Prostate cancer \\
\hline Mesothelin & $\begin{array}{l}\text { - DMOT4039A [9] } \\
\text { - Anetumab ravtensine (BAY-94-9343) [10] }\end{array}$ & Ovarian, pancreatic cancer ar \\
\hline Nectin 4 & $\begin{array}{l}\text { - Enfortumab vedotin (ASG-22M6E) [11] } \\
\text { - } \mathrm{ASC}-22 \mathrm{CE}[12]\end{array}$ & Bladder, breast, lung and par \\
\hline ENPP3 & AGS-16M8F [13] & Renal cell carcinoma, liver ca \\
\hline Guanylyl cyclase C (GCC) & Indusatumab vedotin (MLN-0264) [14] & Pancreatic and colorectal can \\
\hline SLC44A4 & ASG-5ME [15] & Pancreatic, gastric and prosta \\
\hline $\mathrm{NaPi} 2 \mathrm{~b}$ & - anti-NaPi2b ADC, Lifastuzumab vedotin [16] & Non-small cell lung cancer ar \\
\hline CD70 (TNFSF7) & $\begin{array}{l}\text { - } \text { DNIB0600A [17] } \\
\text { - } \text { AMG-172 [18] } \\
\text { - } \text { MDX-1203 [19] } \\
\text { - Vorsetuzumab mafodotin SGN-75 [20] }\end{array}$ & Renal cell carcinoma \\
\hline CA9, Carbonic anhydrase & BAY79-4620 [21] & Solid tumours \\
\hline $5 T 4$ (TPBG) & PF 06263507 [22] & Solid tumours \\
\hline SLTRK6 & ASG-15ME [23] & Bladder cancer \\
\hline SC-16 (anti-Fyn3) & SC16LD6.5 [24] & $\begin{array}{l}\text { SCLC } \\
\text { NSCLC and ovarian cancer }\end{array}$ \\
\hline Tissue factor & HuMax-TF-ADC (TF-011-MMAE) [25] & Solid tumours \\
\hline LIV-1 (ZIP6) & SGN-LIV1A [26] & Breast cancer \\
\hline P-Cadherin & PCA062 [27] & Solid tumours \\
\hline PSMA & $\begin{array}{ll}\text { - } & \text { MLN2704 [28] } \\
\text { - } & \text { PSMA-ADC [29] }\end{array}$ & Prostate cancer \\
\hline
\end{tabular}

Target antigens in the tumour vasculature and stroma

(ED-B)

Endothelin receptor ETB

VEGFR2 (CD309)

Tenascin C

Collagen IV

Periostin

Human mAb L19 and F8 [30]

RG-7636 [31]

Anti-VEGFR-2ScFv-As2O3-stealth Nanoparticles [32]

Melanoma

Solid tumours

Solid tumours

Solid tumours

Cytotoxic immunoconjugates [34]

Solid tumours

Target antigens regulated from driver oncogenes

\begin{tabular}{l|l}
\hline HER 2 & $\bullet$ T-DM1[36] \\
& $\bullet A R X 788[37]$ \\
EGFR & $\bullet$ SYD985 [38] \\
& $\bullet$ ABT-414 [39] \\
& $\bullet$ IMGN289 [40] \\
& $\bullet M G-595[41]$
\end{tabular}

Breast cancer

Glioblastoma, NSCLC, head and neck, breast, oesophageal

Target antigens in haematological malignancies

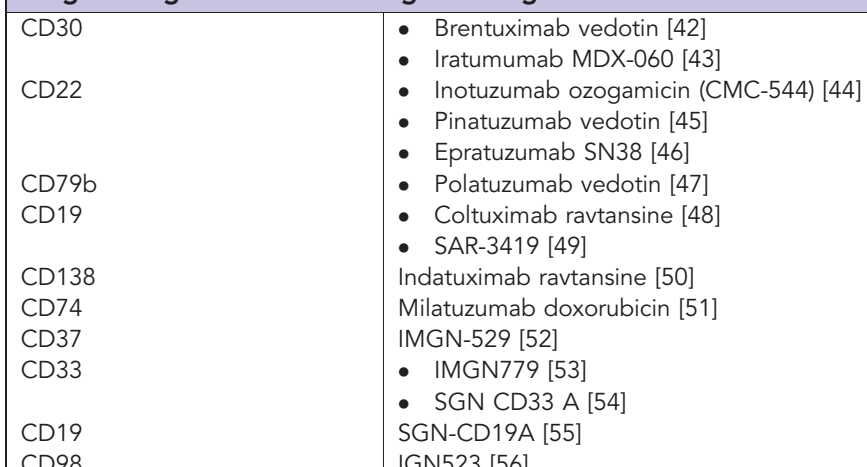

$\mathrm{HL}$ and $\mathrm{ALCL}$
$\mathrm{NHL}$ and $\mathrm{ALL}$
$\mathrm{DLBCL}$ and follicular NHL
DLBCL and ALL
Multiple myeloma
$\mathrm{CLL}, \mathrm{NHL}$ and multiple myeloma
$\mathrm{NHL}$ and $\mathrm{CLL}$
$\mathrm{AML}$
$\mathrm{ALL}$ and $\mathrm{NHL}$,
$\mathrm{AML}$

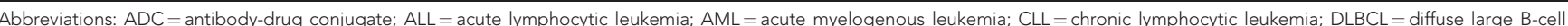
lymphoma; $\mathrm{HL}=$ Hodgkin lymphoma; $\mathrm{NHL}=$ non-Hodgkin lymphoma; NSCLC = non-small cell lung cancer; $\mathrm{SCLC}=$ small cell lung cancer; $\mathrm{TNBC}=$ triple-negative breast cancer. Please refer to the Supplementary Data File for all references cited in this table.

consist of an undesirable heterogeneous mixture of ADCs containing drug molecules with high DAR. There are ongoing efforts for more homogeneous ADCs with increased number of drug molecules stably linked to the Ab. The optimal DAR is undetermined and highly dependent on other ADC variables; however, more commonly the ADCs aim to attain a DAR close to 4. (Hamblett et al, 2004; Teicher and Chari, 2011). Site-specific conjugation, has been an important step in ADC development, enabled the production of homogeneous ADCs with the desired and prespecified DAR by using techniques such as engineered cysteine 
residue, non-canonical amino acid incorporation or modification of peptide tags (Panowksi et al, 2014; Agarwal and Bertozzi, 2015).

Linkers can be divided in non-cleavable and cleavable. Noncleavable linkers have the characteristic that following ADC lysosomal degradation the cytotoxic payload remains active while still being attached to the linker and an amino acid residue. T-DM1 is an ADC that uses a non-cleavable linker (Junttila et al, 2011; Shefet-Carasso and Benhar, 2015).

Cleavable linkers use different methods to release cytotoxic drug, increasing the possibility of the bystander effect. There are acidsensitive linkers that will release the free drug in the low $\mathrm{pH}$ conditions in lysosomes or endosomes. Such a mechanism was used by gemtuzumab ozogamicin and its linker was characterised by certain plasma instability (Hamann et al, 2002; Shefet-Carasso and Benhar, 2015). Inotuzumab ozogamicin, now in phase II trials, uses the same linker and it is more stable (Boghaert et al, 2008). Another type of cleavable linker are lysosomal protease-sensitive linkers with the licensed example of brentuximab vedotin (Senter and Sievers, 2012). A third type are glutathione-sensitive linkers that benefit from the higher concentration of glutathione in tumour cells. (Sapra et al, 2011). Each approach has different advantages and disadvantages, so the choice of the linker can only be determined by taking into account all the other components of the ADC.

Cytotoxic payload. To create an effective ADC, it is imperative to have a potent cytotoxic payload. The first generation of ADCs used classical chemotherapy drugs such as doxorubicin and methotrexate with the benefit of a well-known toxicity profile (Shefet-Carasso and Benhar, 2015). Repeated studies, however, have shown that the actual concentration of the cytotoxic payload in tumour cells is minimal with only $1-2 \%$ of the administered dose reaching the tumour (Teicher and Chari, 2011). It is evident that the optimal chemotherapy drug used should be extremely potent, being effective at picomolar or nanomolar concentrations.

There are two main categories of cytotoxic drugs used in ADC development: microtubule inhibitors and DNA-damaging drugs. Auristatins block tubulin assembly and cause G2/M phase cell cycle arrest; they are the most commonly used payloads, accounting for a majority of cytotoxic payloads used in ADCs currently investigated (Sapra and Shor, 2013; Bouchard et al, 2014). Monomethyl auristatin E, an auristatin derivative (MMAE) is the cytotoxic payload of brentuximab vendotin and has a free drug $\mathrm{IC}_{50}: 10^{-11}-10^{-9}$ allowing it to be effective in the low nanomolar range (Gerber et al, 2009). Maytansinoids, another class of tubulin inhibitors, have also been used successfully in the ADC development. The cytotoxic payload of trastuzumab emtansine (T-DM1) DM1 is a highly potent maytansinoid, developed by Immunogen, with a free drug $\mathrm{IC}_{50}: 10^{-11}-10^{-9}$. Another maytansinoid drug, DM4 incorporated on SAR3419, is being investigated in phase II trials exhibiting significant 'bystander effect' as the ADC's metabolites have lipophilic properties allowing crossing of the cellular membrane (Blanc et al, 2011). Tubulysins are a promising new class of tubulin inhibitors. Tubulisin analogues were successfully conjugated to trastuzumab forming a stable and potent ADC (Cohen et al, 2014). The DNA-damaging agents have the ability to be active throughout the different cell cycle phases. Duocarmycin is a powerful cytotoxic alkylating compound that binds to the minor groove of DNA and has shown activity against various multidrug-resistant models. Duocarmycin-based ADCs are currently under investigation in a phase 1 trial setting conjugated to the anti-HER-2 antibody, trastuzumab (Van Herpen et al, 2015).

Calicheamicin is a potent antitumour antibiotic that causes double-strand DNA breaks and rapid cell death by binding to the DNA's minor groove. It is less dependent on cell cycle progression making it potentially useful against TICs who have lower rates of proliferation (Gupta et al, 2009; Sapra et al, 2011). Gemtuzumab ozogamicin and other ADCs such as inotuzumab ozogamicin in
non-Hodgkin lymphoma and MDX-1203 in renal cancer are using these agents (Owonikoko et al, 2014).

A potential new drug under investigation is $\alpha$-amanitin, an RNA polymerase II inhibitor in the picomolar range, derived from the mushroom, Amanita phalloides (Moldenhauer et al, 2012). Other topoisomerase inhibitors under investigation is the SN38, the active metabolite of irinotecan (Palakurthi, 2015). Another new category is the pyrrolobenzodiazepines (PBDs) that bind to discrete DNA sequences causing lethal lesions and have interestingly not been found to have cross-resistance with common chemotherapeutic agents (Bouchard et al, 2014).

A different approach, but in line with ADC principles, are the radioimmunoconjugates where radionuclides are the cytotoxic payloads linked to monoclonal antibodies. Two radioimmunoconjugates targeting CD20 have been approved for treatment of refractory HodHL, (Yttrium-90-ibritumomab tiuxetan and iodine131-tositumomab). Despite its efficacy due to under-utilisation tositumomab manufacturer has discontinued its production. In solid tumours, radioimmunoconjugates are being investigated, particularly in treating minimal residual disease in prostate and colorectal cancer among others (Kraeber-Bodere et al, 2014).

\section{CLINICAL EXPERIENCE}

T-DM1, Kadcyla. The only ADC licensed in non-haematological malignancies is ado-trastuzumab-emtansine (T-DM1, Kadcyla, Roche, Genentech), the antibody used is the well-known trastuzumab, a humanised IgG1 anti-HER-2 Ab linked with a stable non-cleavable linker to the maytansinoid DM1 (LoRusso et al, 2011; Verma et al, 2012). T-DM1 has been approved in the second line setting by the FDA in 2013 for HER-2-positive patients who had previously received treatment with trastuzumab and taxane chemotherapy. T-DM1 has an overall tolerable toxicity profile with most common adverse events being fatigue, transaminitis, nausea, thrombocytopenia and rash. In USA, T-DM1 carries black box warnings for hepatotoxicity, embryo-fetal and cardiac toxicity $P<0.001$ ) (Verma et al, 2012).

T-DM1 is also being investigated as a single agent comparted to docetaxel in previously treated gastric cancer with results expected in 2015 (GATSBY trial, NCT01641939) (Shah, 2015).

Brentuximab vedotin, Adcetris. Brentuximab vedotin (BV, Adcetris, Seattle Genetics) is composed of an anti-CD30 mAb connected with a cleavable peptide to the highly potent tubulin inhibitor MMAE discussed previously. CD30 is a member of the tumour necrosis factor (TNF) family identified on Reed-Sternberg cells of classical Hodgkin lymphoma (HL). Binding of BV to the cell surface will lead to internalisation and lysosomal proteolytic cleavage of the linker releasing the MMAE (Senter and Sievers, 2012; Sievers and Senter, 2013).

BV has gained approval for the treatment of patients with relapsed or refractory $\mathrm{CD} 30+\mathrm{HL}$ following autologous stem cell transplant (ASCT) or patients not legible for ASCT who have failed at least two other chemotherapy treatments. BV has also been approved for patients with anaplastic large cell lymphoma (ALCL) as a second line. The accelerated approval for Hodgkin's lymphoma was based on a single-arm phase II clinical trial, where there was a $73 \%$ response rate, $32 \%$ complete remission and a median duration 20.5 months (Younes et al, 2012). The indication for ALCL was established based on the impressive results of the phase II study. Patients at this study had an $86 \%$ overall response rate and 54\% complete responses (Pro et al, 2012). The most common adverse reactions were peripheral sensory neuropathy, neutropenia, fatigue, nausea and thrombocytopenia. In USA, BV carries a black box warning for progressive multifocal leukoencephalopathy (Younes et al, 2012). 
Gemtuzumab ozogamicin, Mylotarg. Gemtuzumab ozoogamicin (GO, Mylotarg, Pfizer) was the first ADC to be approved by the FDA in 2000. It was licensed as a monotherapy in patients over the age of 60 with acute myelogenous leukemia (AML) who were not candidates for cytotoxic chemotherapy. GO consisted of a humanised IgG4 mAb directed against CD33 a surface antigen present in 85-90\% of AML linked to a calicheamicin cytotoxin with positive results coming from the first single-agent phase II studies achieving 30\% remissions (Tsimberidou et al, 2006). In 2010, the results of a post-approval phase III study showed no clinical benefit. In fact, the patients in the GO arm had a higher risk of fatal AEs and this trial led to the retraction of its FDA licence approval (Petersdorf et al, 2013). The failures were attributed to an unstable linker allowing premature release of the cytotoxic payload and to the notso-selective antibody target (ten Cate et al, 2009).

More recent trials such as the ALFA-0701 using intermittent dosing regimens have showed good results, improving event-free survival and overall survival in patients with AML, reigniting discussions about the future of GO (Castaigne et al, 2012).

Clinical trial setting. Research in ADCs has been very active with almost 120 total clinical trials with the majority focusing on haematological malignancies. Interest in solid tumours is evident with 52 open phase I/II studies There are approximately 50 unique ADCs under investigation, with 35 ADCs investigated in solid tumours (Mullard, 2013).

Putative mechanisms of resistance. Cancer cells under the pressure of any treatment develop resistance mechanisms that will allow them to survive. These mechanisms are complex and variable confronting the $\mathrm{ADC}$ in each of its different components, the cytotoxic drug, the monoclonal antibody or by activating survival signalling pathways (Shefet-Carasso and Benhar, 2015).

The cytotoxic payload of the ADC is subject to the same multidrug resistance mechanisms as conventional chemotherapy drugs. Some of the most commonly used drugs in the new generation ADCs such as auristatins (MMAE) and calicheamicin are substrates of P-glycoprotein. Resistance can also emerge from mechanisms that will limit the intracellular concentration of the ADC. Barok et al (2014) have described such mechanisms in their work with T-DM1, that is, downregulation of the target antigen, reduced internalisation of the ADC, diminished lysosomal degradation or increased ADC recycling to the cell surface and masking of the antigen epitope. There is also evidence that activation of the PI3K/AKT, MEK/ERK and JAK/STAT pathways leads to increased ADC resistance indicating that a potential way ahead could be combinations with small molecule inhibitors (Shefet-Carasso and Benhar, 2015). Clonal expansion of resistant cells and intratumoural heterogeneity representing populations of cells that are resistant de novo within the same tumour, are likely to influence resistance to ADCs (Gerlinger et al, 2012).

\section{CONCLUSION: FUTURE PROSPECTS}

Predictive biomarkers are essential to ensure we are offering the most effective treatments to the group of patients most likely to benefit from them. Development of specific tests that will determine the chances of responding to treatment to an ADC by investigating the level of expression of the target antigen similar to the immunohistochemical (IHC) confirmation of HER-2 expression are already being explored (Mack et al, 2014). Efforts are ongoing in order to move away from IHC-based techniques that require tissue biopsy and use circulating tumour cells or imaging techniques to identify the patient population more likely to respond (Mack et al, 2014).

Another approach to ADCs is to use high-affinity molecules like folic acid or growth hormones as cytotoxic payload carriers or small antibody fragments such as diabodies or minibodies. The rationale behind this approach is that it might result in more effective drug delivery and increased antitumour activity. However, this approach will also limit the pharmacokinetic benefits of an ADC resulting in more rapid clearance and larger volume of distribution.

Combination strategies are actively explored in many ongoing clinical trials. T-DM1 is being investigated in combination with pertuzumab (Phillips et al, 2014). Other combinations of ADCs investigated are with conventional chemotherapies, for example, brentuximab with modified AVD, PI3K inhibitors like BYL719 with T-DM1 or with TKI inhibitors like neratinib.

There remain major hurdles that ADCs need to overcome: low delivery efficiency, target antigens expressed in normal tissues, the heterogeneity of target antigen expression in the tumour and more (Teicher and Chari, 2011). The future of ADCs seems promising as the combination of new linker technologies and more powerful cytotoxic payloads leads to the emergence of more stable and effective ADCs.

\section{ACKNOWLEDGEMENTS}

The Drug Development Unit of the Royal Marsden NHS Foundation Trust and The Institute of Cancer Research is supported in part by a programme grant from Cancer Research UK. Support is also provided by the Experimental Cancer Medicine Centre (programme grant) (to The ICR) and the National Institute for Health Research Biomedical Research Centre (jointly awarded to the RMH NHS Foundation Trust and The ICR). Scholarship awarded by the Hellenic Society of Medical Oncology (HESMO).

\section{CONFLICT OF INTEREST}

The authors declare no conflict of interest.

\section{REFERENCES}

Agarwal P, Bertozzi CR (2015) Site-specific antibody-drug conjugates: the nexus of bioorthogonal chemistry, protein engineering, and drug development. Bioconjug Chem 26(2): 176-192.

Barok M, Joensuu H, Isola J (2014) Trastuzumab emtansine: mechanisms of action and drug resistance. Breast Cancer Res 16(2): 209.

Baselga J, Verma S, Ro J, Huober J, Guardino E, Fang L, Olsen S, Phillips GL, de Haas S, Pegram M (2013) Relationship between tumor biomarkers and efficacy in EMILIA, a phase III study of trastuzumab emtansine (T-DM1) in HER2-positive metastatic breast cancer. AACR Annual Meeting. Abstract LB-63. Presented April 7, 2013.

Blanc V, Bousseau A, Caron A, Carrez C, Lutz RJ, Lambert JM (2011) SAR3419: an anti-CD19-Maytansinoid immunoconjugate for the treatment of B-cell malignancies. Clin Cancer Res 17(20): 6448-6458.

Boghaert ER, Khandke KM, Sridharan L, Dougher M, DiJoseph JF, Kunz A, Hamann PR, Moran J, Chaudhary I, Damle NK (2008) Determination of pharmacokinetic values of calicheamicin-antibody conjugates in mice by plasmon resonance analysis of small (5 microl) blood samples. Cancer Chemother Pharmacol 61(6): 1027-1035.

Bouchard H, Viskov C, Garcia-Echeverria C (2014) Antibody-drug conjugates-a new wave of cancer drugs. Bioorg Med Chem Lett 24(23): 5357-5363.

Casi G, Neri D (2012) Antibody-drug conjugates: basic concepts, examples and future perspectives. J Control Release 161(2): 422-428.

Castaigne S, Pautas C, Terre C, Raffoux E, Bordessoule D, Bastie JN, Legrand O, Thomas X, Turlure P, Reman O, de Revel T, Gastaud L, de Gunzburg N, Contentin N, Henry E, Marolleau JP, Aljijakli A, Rousselot P, Fenaux P, Preudhomme C, Chevret S, Dombret H, Acute Leukemia French A (2012) Effect of gemtuzumab ozogamicin on survival of adult patients with de-novo acute myeloid leukaemia (ALFA-0701): a randomised, open-label, phase 3 study. Lancet 379(9825): 1508-1516.

Cohen R, Vugts DJ, Visser GW, Stigter-van Walsum M, Bolijn M, Spiga M, Lazzari P, Shankar S, Sani M, Zanda M, van Dongen GA (2014) Development 
of novel ADCs: conjugation of tubulysin analogues to trastuzumab monitored by dual radiolabeling. Cancer Res 74(20): 5700-5710.

Flygare JA, Pillow TH, Aristoff P (2013) Antibody-drug conjugates for the treatment of cancer. Chem Biol Drug Des 81(1): 113-121.

Gerber HP, Kung-Sutherland M, Stone I, Morris-Tilden C, Miyamoto J, McCormick R, Alley SC, Okeley N, Hayes B, Hernandez-Ilizaliturri FJ, McDonagh CF, Carter PJ, Benjamin D, Grewal IS (2009) Potent antitumor activity of the anti-CD19 auristatin antibody drug conjugate hBU12-vcMMAE against rituximab-sensitive and -resistant lymphomas. Blood 113(18): 4352-4361.

Gerlinger M, Rowan AJ, Horswell S, Larkin J, Endesfelder D, Gronroos E, Martinez P, Matthews N, Stewart A, Tarpey P, Varela I, Phillimore B, Begum S, McDonald NQ, Butler A, Jones D, Raine K, Latimer C, Santos CR, Nohadani M, Eklund AC, Spencer-Dene B, Clark G, Pickering L, Stamp G, Gore M, Szallasi Z, Downward J, Futreal PA, Swanton C (2012) Intratumor heterogeneity and branched evolution revealed by multiregion sequencing. $N$ Engl J Med 366(10): 883-892.

Gupta PB, Chaffer CL, Weinberg RA (2009) Cancer stem cells: mirage or reality? Nat Med 15(9): 1010-1012.

Hamann PR, Hinman LM, Hollander I, Beyer CF, Lindh D, Holcomb R, Hallett W, Tsou HR, Upeslacis J, Shochat D, Mountain A, Flowers DA, Bernstein I (2002) Gemtuzumab ozogamicin, a potent and selective antiCD33 antibody-calicheamicin conjugate for treatment of acute myeloid leukemia. Bioconjug Chem 13(1): 47-58.

Hamblett KJ, Senter PD, Chace DF, Sun MM, Lenox J, Cerveny CG, Kissler KM, Bernhardt SX, Kopcha AK, Zabinski RF, Meyer DL, Francisco JA (2004) Effects of drug loading on the antitumor activity of a monoclonal antibody drug conjugate. Clin Cancer Res 10(20): 7063-7070.

Hughes B (2010) Antibody-drug conjugates for cancer: poised to deliver? Nat Rev Drug Discov 9(9): 665-667.

Junttila TT, Li G, Parsons K, Phillips GL, Sliwkowski MX (2011) Trastuzumab-DM1 (T-DM1) retains all the mechanisms of action of trastuzumab and efficiently inhibits growth of lapatinib insensitive breast cancer. Breast Cancer Res Treat 128(2): 347-356.

Kraeber-Bodere F, Bodet-Milin C, Rousseau C, Eugene T, Pallardy A, Frampas E, Carlier T, Ferrer L, Gaschet J, Davodeau F, Gestin JF, Faivre-Chauvet A, Barbet J, Cherel M (2014) Radioimmunoconjugates for the treatment of cancer. Semin Oncol 41(5): 613-622.

LoRusso PM, Weiss D, Guardino E, Girish S, Sliwkowski MX (2011) Trastuzumab emtansine: a unique antibody-drug conjugate in development for human epidermal growth factor receptor 2-positive cancer. Clin Cancer Res 17(20): 6437-6447.

Mack F, Ritchie M, Sapra P (2014) The next generation of antibody drug conjugates. Semin Oncol 41(5): 637-652.

Moldenhauer G, Salnikov AV, Luttgau S, Herr I, Anderl J, Faulstich H (2012) Therapeutic potential of amanitin-conjugated anti-epithelial cell adhesion molecule monoclonal antibody against pancreatic carcinoma. J Natl Cancer Inst 104(8): 622-634.

Mullard A (2013) Maturing antibody-drug conjugate pipeline hits 30. Nat Rev Drug Discov 12(5): 329-332.

Owonikoko TK, Hussain A, Stadler WM, Smith DC, Sznol M, Molina AM, Gulati P, Shah A, Ahlers CM, Cardarelli J, Cohen LJ (2014) A Phase I multicenter open-label dose-escalation study of BMS-936561 (MDX-1203) in clear cell renal cell carcinoma (ccRCC) and B-cell non Hodgkin lymphoma (B-NHL). J Clin Oncol 32: 5s (suppl; abstr 2558).

Palakurthi S (2015) Challenges in SN38 drug delivery: current success and future directions. Expert Opin Drug Deliv 27: 1-11.

Palumbo A, Hauler F, Dziunycz P, Schwager K, Soltermann A, Pretto F, Alonso C, Hofbauer GF, Boyle RW, Neri D (2011) A chemically modified antibody mediates complete eradication of tumours by selective disruption of tumour blood vessels. Br J Cancer 104(7): 1106-1115.

Panowksi S, Bhakta S, Raab H, Polakis P, Junutula JR (2014) Site-specific antibody drug conjugates for cancer therapy. MAbs 6(1): 34-45.

Perez HL, Cardarelli PM, Deshpande S, Gangwar S, Schroeder GM, Vite GD, Borzilleri RM (2014) Antibody-drug conjugates: current status and future directions. Drug Discov Today 19(7): 869-881.

Perrino E, Steiner M, Krall N, Bernardes GJ, Pretto F, Casi G, Neri D (2014) Curative properties of noninternalizing antibody-drug conjugates based on maytansinoids. Cancer Res 74(9): 2569-2578.
Petersdorf SH, Kopecky KJ, Slovak M, Willman C, Nevill T, Brandwein J, Larson RA, Erba HP, Stiff PJ, Stuart RK, Walter RB, Tallman MS, Stenke L, Appelbaum FR (2013) A phase 3 study of gemtuzumab ozogamicin during induction and postconsolidation therapy in younger patients with acute myeloid leukemia. Blood 121(24): 4854-4860.

Phillips GD, Fields CT, Li G, Dowbenko D, Schaefer G, Miller K, Andre F, Burris 3rd HA, Albain KS, Harbeck N, Dieras V, Crivellari D, Fang L, Guardino E, Olsen SR, Crocker LM, Sliwkowski MX (2014) Dual targeting of HER2-positive cancer with trastuzumab emtansine and pertuzumab: critical role for neuregulin blockade in antitumor response to combination therapy. Clin Cancer Res 20(2): 456-468.

Pro B, Advani R, Brice P, Bartlett NL, Rosenblatt JD, Illidge T, Matous J, Ramchandren R, Fanale M, Connors JM, Yang Y, Sievers EL, Kennedy DA, Shustov A (2012) Brentuximab vedotin (SGN-35) in patients with relapsed or refractory systemic anaplastic large-cell lymphoma: results of a phase II study. J Clin Oncol 30(18): 2190-2196.

Sapra P, Damelin M, Dijoseph J, Marquette K, Geles KG, Golas J, Dougher M, Narayanan B, Giannakou A, Khandke K, Dushin R, Ernstoff E, Lucas J, Leal M, Hu G, O'Donnell CJ, Tchistiakova L, Abraham RT, Gerber HP (2013) Long-term tumor regression induced by an antibody-drug conjugate that targets 5T4, an oncofetal antigen expressed on tumorinitiating cells. Mol Cancer Ther 12(1): 38-47.

Sapra P, Hooper AT, O’Donnell CJ, Gerber HP (2011) Investigational antibody drug conjugates for solid tumors. Expert Opin Invest Drugs 20(8): 1131-1149.

Sapra P, Shor B (2013) Monoclonal antibody-based therapies in cancer: advances and challenges. Pharmacol Ther 138(3): 452-469.

Senter PD, Sievers EL (2012) The discovery and development of brentuximab vedotin for use in relapsed Hodgkin lymphoma and systemic anaplastic large cell lymphoma. Nat Biotechnol 30(7): 631-637.

Shah MA (2015) Update on metastatic gastric and esophageal cancers. J Clin Oncol 33(16): 1760-1769.

Shefet-Carasso L, Benhar I (2015) Antibody-targeted drugs and drug resistance-Challenges and solutions. Drug Resist Updat 18: 36-46.

Sievers EL, Senter PD (2013) Antibody-drug conjugates in cancer therapy. Ann Rev Med 64: 15-29.

Teicher BA, Chari RV (2011) Antibody conjugate therapeutics: challenges and potential. Clin Cancer Res 17(20): 6389-6397.

ten Cate B, Bremer E, de Bruyn M, Bijma T, Samplonius D, Schwemmlein M, Huls G, Fey G, Helfrich W (2009) A novel AML-selective TRAIL fusion protein that is superior to Gemtuzumab Ozogamicin in terms of in vitro selectivity, activity and stability. Leukemia 23(8): 1389-1397.

Tsimberidou AM, Giles FJ, Estey E, O’Brien S, Keating MJ, Kantarjian HM (2006) The role of gemtuzumab ozogamicin in acute leukaemia therapy. Br J Haematol 132(4): 398-409.

Van Herpen CM, Banerji U, Mommers EC, Koper NP, Goedings P, Lopez J, Awada A, Fiebrich HB, Aftimos PG (2015) Phase I Dose-Escalation Trial with the DNA-Alkylating Anti-HER2 Antibody-Drug Conjugate SYD985. European Cancer Congress: Vienna, Austria.

Verma S, Miles D, Gianni L, Krop IE, Welslau M, Baselga J, Pegram M, Oh DY, Dieras V, Guardino E, Fang L, Lu MW, Olsen S, Blackwell K, Group ES (2012) Trastuzumab emtansine for HER2-positive advanced breast cancer. N Engl J Med 367(19): 1783-1791.

Visvader JE, Lindeman GJ (2008) Cancer stem cells in solid tumours: accumulating evidence and unresolved questions. Nat Rev Cancer 8(10): 755-768.

Younes A, Gopal AK, Smith SE, Ansell SM, Rosenblatt JD, Savage KJ, Ramchandren R, Bartlett NL, Cheson BD, de Vos S, Forero-Torres A, Moskowitz CH, Connors JM, Engert A, Larsen EK, Kennedy DA, Sievers EL, Chen R (2012) Results of a pivotal phase II study of brentuximab vedotin for patients with relapsed or refractory Hodgkin's lymphoma. J Clin Oncol 30(18): 2183-2189.

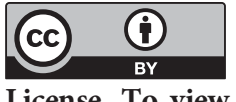

This work is licensed under the Creative Commons Attribution 4.0 International License. To view a copy of this license, visit http://creativecommons.org/licenses/by/4.0/

Supplementary Information accompanies this paper on British Journal of Cancer website (http://www.nature.com/bjc) 